\title{
MINIMAL CLONES GENERATED BY MAJORITY OPERATIONS
}

\author{
TAMÁS WALDHAUSER
}

Abstract. The minimal majority functions of the four-element set are determined.

\section{INTRODUCTION}

A set $C$ of finitary operations on a set $A$ is a clone if it is closed under composition of functions and contains all projections. In this paper we shall be concerned only with clones on a finite set.

The set of all finitary operations on $A$ is a clone as well as the set of all projections. These are the largest and the smallest clones on $A$, the latter is often called the trivial clone. The clone generated by a set $F$ of finitary functions on $A$ is the intersection of all clones containing $F$, i.e. the smallest clone containing $F$. This clone is denoted by $[F]$. If $F=\{f\}$ then we simply write $[f]$. Clearly $[F]$ consists of those functions which can be obtained from the elements of $F$ and from the projections by a finite number of compositions. In other words, $[F]$ is the set of term functions of the algebra $\langle A, F\rangle$.

We say that the $n$-ary function $f$ preserves the relation $\rho \subseteq A^{k}$ if for all $a_{i, j} \in A$ $(i=1, \ldots, k, j=1, \ldots, n)$

$$
\left(a_{11}, a_{21}, \ldots, a_{k 1}\right),\left(a_{12}, a_{22}, \ldots, a_{k 2}\right), \ldots,\left(a_{1 n}, a_{2 n}, \ldots, a_{k n}\right) \in \rho
$$

implies

$$
\left(f\left(a_{11}, a_{12}, \ldots, a_{1 n}\right), f\left(a_{21}, a_{22}, \ldots, a_{2 n}\right), \ldots, f\left(a_{k 1}, a_{k 2}, \ldots, a_{k n}\right)\right) \in \rho .
$$

Preserving a relation is inherited when composing functions:

$$
\text { If } f \text { preserves a relation } \rho \text { and } g \in[f] \text {, then } g \text { also preserves } \rho \text {. }
$$

An important special case is that of unary relations: $f$ preserves $B \subseteq A$ iff $B$ is closed under $f$. If $f$ preserves all subsets of $A$ then we say that $f$ is conservative (cf. [5]).

A clone is minimal if it has no proper subclones except for the trivial one. On finite sets every clone contains a minimal one (cf. 6]). Obviously a clone is minimal iff it is generated by every nontrivial member of it. (By a nontrivial function we mean a function which is not a projection.) If $C$ is a minimal clone and $f$ is nontrivial, and of minimum arity in $C$ then we say that $f$ is a minimal function (cf. 7] p.408)

By a theorem of I. G. Rosenberg [7, every minimal clone (on a finite set) is generated by a nontrivial minimal function $f$, for which one of the following holds:

1) $f$ is unary and $f^{2}(x) \approx f(x)$ or $f^{p}(x) \approx x$ for some prime $p$.

2) $f$ is binary idempotent, i.e. $f(x, x) \approx x$.

3) $f$ is ternary majority, i.e. $f(x, x, y) \approx f(x, y, x) \approx f(y, x, x) \approx x$.

4) $f$ is a semiprojection, i.e. there exists an $i$ such that $f\left(x_{1}, x_{2}, \ldots, x_{n}\right)=x_{i}$ whenever the arguments are not pairwise distinct.

5) $f(x, y, z)=x+y+z$ where $\langle A,+\rangle$ is a Boolean group.

2000 Mathematics Subject Classification. 08A40.

Key words and phrases. Clones, majority operations.

This research was supported by Ministry of Culture and Education of Hungary, grant No. FKFP 0877/1997. 
Note that in each case $f$ cannot generate a nontrivial function which is of lesser arity than $f$. This means that $f$ is a minimal function iff $[f]$ is a minimal clone. In cases 1) and 5) the conditions ensure the minimality of $f$, while in the other cases they do not.

In 4. Post described all clones on a two-element set, in [1] B. Csákány determined the minimal clones of a three-element set. For the four-element case binary minimal clones were described by B. Szczepara in 8 .

Conservative minimal majority and binary functions were determined on any finite set by B. Csákány in [2].

In this paper we prove the following description of all the minimal majority functions of a four-element set.

Theorem 1.1. If $C$ is a minimal clone on a four-element set $A$ and it contains a majority function, then $C=[f]$ where $f$ is either conservative or $\langle A, f\rangle \cong$ $\left\langle\{1,2,3,4\}, M_{i}\right\rangle$ for some $i \in\{1,2,3\}$ (see the table below).

\begin{tabular}{|c|ccc|}
\hline & $M_{1}$ & $M_{2}$ & $M_{3}$ \\
\hline$(1,2,3)$ & 4 & 4 & 3 \\
$(2,3,1)$ & 4 & 2 & 3 \\
$(3,1,2)$ & 4 & 3 & 3 \\
\hline$(2,1,3)$ & 4 & 2 & 4 \\
$(1,3,2)$ & 4 & 4 & 4 \\
$(3,2,1)$ & 4 & 3 & 4 \\
\hline$\{1,2,4\}$ & 4 & 4 & 4 \\
\hline$\{1,3,4\}$ & 4 & 4 & 4 \\
\hline$(4,2,3)$ & 4 & 4 & 3 \\
$(2,3,4)$ & 4 & 2 & 3 \\
$(3,4,2)$ & 4 & 3 & 3 \\
\hline$(2,4,3)$ & 4 & 2 & 4 \\
$(4,3,2)$ & 4 & 4 & 4 \\
$(3,2,4)$ & 4 & 3 & 4 \\
\hline
\end{tabular}

The middle two rows mean that if $\{a, b, c\}=\{1,2,4\}$ or $\{1,3,4\}$, then $M_{i}(a, b, c)=4$ for $i=1,2,3$. For the triplets not listed in the table the majority rule defines the value of the functions.

\section{MAJORITY FUnCTIONS ON Finite SETS}

If $C$ is a clone which is generated by a majority function then we shall briefly say that $C$ is a majority clone.

Let $A$ be a finite set and $f$ be a majority function on $A$. We define the range of $f$ in the following way:

$$
\text { range }(f)=\{f(a, b, c) \mid a, b, c \in A \text { are pairwise distinct }\} .
$$

A simple induction argument shows that if $g$ is a nontrivial function in a majority clone, then $g$ is a so-called near-unanimity function, i.e.

$$
g(y, x, x, \ldots, x, x) \approx g(x, y, x, \ldots, x, x) \approx \ldots \approx g(x, x, x, \ldots, x, y) \approx x
$$

(cf. (7) of [2]).

In Rosenberg's theorem $f$ cannot be a near-unanimity function except for the majority case, so any minimal subclone of a majority clone is again a majority clone. This means that in order to prove the minimality of a majority clone $C$, it suffices to show that any two majority functions in $C$ generate each other.

To show the nonminimality of a clone $[f]$ we will make use of the following facts.

Lemma 2.1. Let $f$ be a majority function on $A$.

(2.1) If $f$ is a minimal function and it preserves $B \subseteq A$ then $\left.f\right|_{B}$ must be a minimal function on $B$.

(2.2) If a nontrivial $g \in[f]$ preserves some $B \subseteq A$ but $f$ does not, then $[f]$ is not minimal. 
(2.3) If the range of some nontrivial $g \in[f]$ does not contain an element which belongs to the range of $f$, then $[f]$ is not minimal. (Cf. Corollary 1.25 of [9.)

Proof.

(2.1) Composing functions and restricting functions commute.

(2.2) This follows from (1.1).

(2.3) This also follows from (1.1) since $a \notin$ range $(g)$ iff $g$ preserves the equivalence relation whose blocks are $\{a\}$ and $A \backslash\{a\}$.

We can use (2.1) for three-element set $B$ because we know the minimal majority clones for such set. These are described in [1] as follows. If $C$ is a minimal majority clone on a three-element set $A$, then there exists an $f \in C$ such that

$$
\langle A, f\rangle \cong\left\langle\{1,2,3\}, m_{i}\right\rangle \text { for some } i=1,2,3,
$$

where $m_{1}, m_{2}, m_{3}$ are the following majority functions.

For $\left\{x_{0}, x_{1}, x_{2}\right\}=\{1,2,3\}$ we have

$$
\begin{aligned}
& m_{1}\left(x_{0}, x_{1}, x_{2}\right)=1 \\
& m_{2}\left(x_{0}, x_{1}, x_{2}\right)=x_{0} \\
& \left.m_{3}\left(x_{0}, x_{1}, x_{2}\right)=x_{i+1} \text { if } x_{i}=1 \text { (subscripts taken modulo } 3\right) . \\
& \text { lo }
\end{aligned}
$$

The clones generated by $m_{1}, m_{2}, m_{3}$ contain 1,3 , and 8 majority functions respectively; these are shown in the following table.

\begin{tabular}{|c|c|ccc|cccccccc|}
\hline & $m_{1}$ & $m_{2}$ & & & $m_{3}$ & & & & & & \\
\hline$(1,2,3)$ & 1 & 1 & 2 & 3 & 2 & 3 & 2 & 2 & 3 & 2 & 3 & 3 \\
$(2,3,1)$ & 1 & 2 & 3 & 1 & 2 & 2 & 2 & 3 & 3 & 3 & 3 & 2 \\
$(3,1,2)$ & 1 & 3 & 1 & 2 & 2 & 2 & 3 & 2 & 3 & 3 & 2 & 3 \\
\hline$(2,1,3)$ & 1 & 2 & 1 & 3 & 3 & 3 & 2 & 3 & 2 & 2 & 3 & 2 \\
$(1,3,2)$ & 1 & 1 & 3 & 2 & 3 & 2 & 3 & 3 & 2 & 3 & 2 & 2 \\
$(3,2,1)$ & 1 & 3 & 2 & 1 & 3 & 3 & 3 & 2 & 2 & 2 & 2 & 3 \\
\hline
\end{tabular}

Conservative minimal majority clones are described in [2] as follows:

If $C$ is a conservative minimal majority clone then there exists an $f \in C$ such that for every three-element $B \subseteq A$ there is an $i_{B} \in\{1,2,3\}$ such that

$$
\left\langle B,\left.f\right|_{B}\right\rangle \cong\left\langle\{1,2,3\}, m_{i_{B}}\right\rangle .
$$

Now we formulate a theorem which helps us reducing the number of functions to be checked, when searching for minimal clones.

Theorem 2.2. Let $f$ be a majority function on a finite set $A$. Then there exists a majority function $g \in[f]$ which satisfies the following identity:

$$
g(g(x, y, z), g(y, z, x), g(z, x, y)) \approx g(x, y, z) .
$$

Proof. We define functions $f^{(k)}(k \geq 1)$ in the following way:

$$
\begin{aligned}
f^{(1)}(x, y, z) & =f(x, y, z), \\
f^{(k+1)}(x, y, z) & =f\left(f^{(k)}(x, y, z), f^{(k)}(y, z, x), f^{(k)}(z, x, y)\right) .
\end{aligned}
$$

We assert that

$$
f^{(k+l)}(x, y, z) \approx f^{(k)}\left(f^{(l)}(x, y, z), f^{(l)}(y, z, x), f^{(l)}(z, x, y)\right)
$$

for $k, l \geq 1$.

This can be proved by induction on $k$; the proof is left to the reader. Let us define a binary operation $*$ on the set $D=\left\{f^{(k)}: k \in \mathbb{N}\right\}$ as follows:

$$
\left(f^{(k)} * f^{(l)}\right)(x, y, z)=f^{(k)}\left(f^{(l)}(x, y, z), f^{(l)}(y, z, x), f^{(l)}(z, x, y)\right) .
$$

The above assertion means that the map $k \mapsto f^{(k)}$ is a homomorphism from $\langle\mathbb{N},+\rangle$ to $\langle D, *\rangle$. So the latter is a finite semigroup, hence it has an idempotent element, say $f^{(k)} * f^{(k)}=f^{(k)}$. And this is just the desired identity for $g=f^{(k)}$. 
Now we introduce some more notation. The function $g=f^{(k)}$ which corresponds to $f$ in the theorem will be denoted by $\widehat{f}$. We put $\langle a b c\rangle=\{(a, b, c),(b, c, a),(c, a, b)\}$, and we will use the symbol $\left.f\right|_{\langle a b c\rangle} \equiv u$ to mean that $f(a, b, c)=f(b, c, a)=$ $f(c, a, b)=u$, and $\left.f\right|_{\langle a b c\rangle}=p$ to mean that $f(a, b, c)=a, f(b, c, a)=b, f(c, a, b)=c$.

The following lemma tells us what identity $*$ means for a majority function.

Lemma 2.3. Let $f$ be a majority function satisfying $(*)$ and let $a, b, c$ be pairwise distinct elements of $A$. Let $u=f(a, b, c), v=f(b, c, a), w=f(c, a, b)$. Then $|\{u, v, w\}| \neq 2$ and if $u, v, w$ are pairwise different, then $\left.f\right|_{\langle u v w\rangle}=p$.

Proof. To prove the first statement, let us suppose (without loss of generality) that $u=v \neq w$. Then (*) for $x=c, y=a, z=b$ yields that $f(w, u, v)=w$, contradicting the majority property of $f$.

The second statement of the lemma follows similarly from $(*)$.

We can say a bit more then Lemma 2.3 when $f$ is a minimal function.

Theorem 2.4. If $f$ is a minimal majority function satisfying (*) and $u=f(a, b, c)$, $v=f(b, c, a), w=f(c, a, b)$ are pairwise different then $\left.f\right|_{\langle u v w\rangle}=p$ and also $\left.f\right|_{\langle v u w\rangle}=p$.

Proof. By the previous lemma we have $\left.f\right|_{\langle u v w\rangle}=p$. Now the nontrivial superposition $g(x, y, z)=f(f(x, y, z), f(x, z, y), x)$ preserves $\{u, v, w\}$ hence $f$ does too, and then from the description of the minimal majority functions on the three-element set we get the conclusion of the theorem.

\section{THE FOUR-ELEMENT CASE}

We have seen that every conservative minimal majority clone is generated by a function $f$ having the following property:

$$
\left.f\right|_{\langle a b c\rangle} \equiv u \text { or }\left.f\right|_{\langle a b c\rangle}=p
$$

for every $a, b, c \in A$ with a suitable $u$ (depending of course, on $a, b, c$ ).

One would hope that it holds for nonconservative clones too. In the first part of this section we are going to try to prove this for a four-element $A$. It will turn out, that the conjecture is not true, but (in the four-element case) there is essentially only one exception. In the second part we determine the minimal ones among the functions satisfying property (3.1), and in the third part we prove the minimality of the clones we have found.

\section{1 .}

Let $S$ denote the set of those majority functions on the set $A=\{1,2,3,4\}$ for which (3.1) holds for any $a, b, c \in A$.

In this section we will show that a minimal majority function which satisfies $(*)$ must belong to the set $S$, or it is isomorphic to $M_{2}$. Since we will consider the values of the functions on the set $\{1,2,3\}$, we introduce one more notation. Let $[p, q, r ; s, t, u]$ denote the set of majority functions $f$ on $A$ for which $f(1,2,3)=p$, $f(2,3,1)=q, f(3,1,2)=r, f(2,1,3)=s, f(1,3,2)=t, f(3,2,1)=u$. If we do not want to specify all these six values of $f$, than we will use $*$ to indicate an arbitrary element of $A$. For example $f \in[4, *, * ; *, *, *]$ means just that $f(1,2,3)=4$. The letters $a, b, c, d$ will always denote arbitrary distinct elements of $A$, i.e. $\{1,2,3,4\}=A=\{a, b, c, d\}$.

First we define and examine a superposition which we will use frequently later on. For a ternary function $f$ let $f_{x}, f_{y}, f_{z}$ stand for the composite functions where the first, second resp. third variable of $f$ is replaced by $f$ itself.

$$
\begin{aligned}
& f_{x}(x, y, z)=f(f(x, y, z), y, z) \\
& f_{y}(x, y, z)=f(x, f(x, y, z), z) \\
& f_{z}(x, y, z)=f(x, y, f(x, y, z))
\end{aligned}
$$


We will briefly write $f_{z y}$ instead of $\left(f_{z}\right)_{y}$. We will also use the convention that lower indices have priority to upper ones. So $f_{z y}^{(2)}$ means $\left(f_{z y}\right)^{(2)}$ and not $\left(f^{(2)}\right)_{z y}$, and also $\widehat{f}_{z y}$ stands for $\widehat{\left(f_{z y}\right)}$.

The proof of the following lemma is just a straightforward calculation, so we omit it.

Lemma 3.1. If $f(a, b, c) \neq d$ then $f_{z y}(a, b, c)=f(a, b, c)$. If this is not the case, then $f_{z y}(a, b, c)=f(a, b, d)$ if the latter does not equal $d$. If it does, then $f_{z y}(a, b, c)=f(a, d, c)$ if it is not $b$. If $f(a, d, c)=b$ then $f_{z y}(a, b, c)=f(a, d, b)$.

From now on $f$ will always denote an arbitrary majority function on $A$, satisfying (*). In the following lemma we prove a nice property of $f$, then through five claims we reach the main result of this section, which is stated in Theorem 3.8. Let us recall that $\langle a b c\rangle$ is just the set $\{(a, b, c),(b, c, a),(c, a, b)\}$, hence $f(\langle a b c\rangle)$ denotes $\{f(a, b, c), f(b, c, a), f(c, a, b)\}$.

Lemma 3.2. If $f$ is minimal and $f(\langle a b c\rangle) \subseteq\{a, b, c\}$ then either $\left.f\right|_{\langle a b c\rangle}=p$ and $\left.f\right|_{\langle b a c\rangle}=p$ or $\left.f\right|_{\langle a b c\rangle} \equiv u$ and $\left.f\right|_{\langle b a c\rangle} \equiv v$ for some $u, v \in A$.

Proof. The set $f(\langle a b c\rangle)$ has three or one elements by Lemma 2.3 . If it has three elements then it is $\{a, b, c\}$, and then by Theorem 2.4 we have $\left.f\right|_{\langle a b c\rangle}=p$ and $\left.f\right|_{\langle b a c\rangle}=p$. In the latter case we may suppose $\left.f\right|_{\langle a b c\rangle} \equiv a$. If $d \notin f(\langle b a c\rangle)$ then $f$ preserves $\{a, b, c\}$ and then the description of the minimal majority functions on the three-element set yields $\left.f\right|_{\langle b a c\rangle} \equiv v$. If $a \in f(\langle b a c\rangle)$ then we permute cyclically the variables to have $f(b, a, c)=a$, and then $g^{(2)}$ preserves $\{a, b, c\}$ for the superposition $g$ of Theorem 2.4. contradicting the minimality of $f$. Finally, if $a \notin f(\langle b a c\rangle)$ but $d \in f(\langle b a c\rangle)$ then $f(\langle b a c\rangle)=\{b, c, d\}$. Now we may suppose $f(b, a, c)=c$, $f(a, c, b)=d, f(c, b, a)=b$ or $f(b, a, c)=b, f(a, c, b)=d, f(c, b, a)=c$ after a cyclic permutation of variables. In the first case $g$, in the second case $g^{(2)}$ shows that $f$ is not minimal, since they preserve $\{a, b, c\}$.

Claim 3.3. In either of the following four cases $f$ is not minimal.

(1) $f \in[4,2,1 ; *, *, *]$

(2) $f \in[4,1,2 ; *, *, *]$

(3) $f \in[4,1,3 ; *, *, *]$

(4) $f \in[4,3,1 ; *, *, *]$

Proof.

(1) Lemma 3.1 shows that $f_{z y}$ preserves $\{1,2,3\}$ (and hence $f$ is not minimal) except when $f(3,2,1)=4, f(3,2,4)=4$ and $f(3,4,1)=4$ or $f(3,4,1)=2, f(3,4,2)=4$. Let us examine the set $f(\langle 213\rangle)$. It has one or three elements by Lemma 2.3 . If it is $\{1\},\{2\}$ or $\{3\}$, then Lemma 3.2 shows that $f$ cannot be minimal. If we have $\left.f\right|_{\langle 213\rangle} \equiv 4$, then we can compute that $f_{z y} \in[1,2,1 ; 2, u, 4]$, where $u \neq 4$. Depending on whether $u=2,3,1$ resp. it can be shown that $\widehat{f}_{z y}$ or $f_{z y}\left(y, z, f_{z y}(x, y, z)\right)$ preserves $\{1,2,3\}$ or $\hat{f}_{z y}$ is not minimal by Lemma 3.2 . Now let us suppose that $f(\langle 213\rangle)$ is a three-element set. If it is $\{1,3,4\}$, then Theorem 2.4 implies $\left.f\right|_{\langle 341\rangle}=p$, hence $f(3,4,1)=3$, but we have seen that it is 4 or 2 . Similarly $f(\langle 213\rangle)=\{1,2,3\},\{2,3,4\}$ is also impossible. So $f(\langle 213\rangle)$ can be nothing else but $\{1,2,4\}$. Since $f(3,2,1)=4$ there are only two possibilities: $f \in[4,2,1 ; 1,2,4]$ or $f \in[4,2,1 ; 2,1,4]$, and then $f_{z y} \in[1,2,1 ; 1,2,4]$ or $f_{z y} \in[1,2,1 ; 2,1,4]$. In both cases Lemma 3.2 yields that $\widehat{f}_{z y}$ is not minimal, hence neither is $f$, and we have finished the proof.

(2) Here we can use the same argument, the only difference is that in this case $f_{z y} \in[1,1,2 ; *, *, *]$.

(3) The function $f(x, z, y)$ is isomorphic to a function which is not minimal by case (1) (We shall note here that changing the second and third variable does not influence the identity (*).) 
(4) Now $f(x, z, y)$ falls under case (2) after renaming the elements of the base set.

Claim 3.4. If $f \in[4,3,2 ; *, *, *]$ then $f$ is not minimal.

Proof. Just as in the previous claim, we examine $f(\langle 213\rangle)$. If it is $\{1\},\{2\},\{3\}$ or $\{1,2,3\}$ then Lemma 3.2 shows that $f$ is not minimal. If $\left.f\right|_{\langle 213\rangle} \equiv 4$ then $g(x, y, z)=f(z, y, f(x, y, z)) \in[3,3,2 ; u, 2, v]$. If none of $u$ and $v$ equals 4 , then $g$ preserves $\{1,2,3\}$. If $u \neq 3$ then $\widehat{h}$ does, for $h(x, y, z)=g(g(x, y, z), z, x)$. Only $u=3, v=4$ remains, but in this case $\widehat{g} \in[3,3,3 ; 2,4,3]$, and it is not a minimal function by Lemma 3.2 . Now let us suppose that $f(\langle 213\rangle)$ is a three-element set containing 4 . If it is $\{1,2,4\}$, then according to Claim 3.3 we must have $f \in[4,3,2 ; 2,1,4]$ or $f \in[4,3,2 ; 1,2,4]$, and in both cases $f_{z y}$ preserves $\{1,2,3\}$. Similarly $f(\langle 213\rangle)=\{1,3,4\}$ implies $f \in[4,3,2 ; 4,1,3]$ or $f \in[4,3,2 ; 4,3,1]$, and again $f_{z y}$ preserves $\{1,2,3\}$. Finally, if $f(\langle 213\rangle)=\{2,3,4\}$ then $f \in[4,3,2 ; 3,4,2]$ or $f \in[4,3,2 ; 2,4,3]$. In the first case $g(x, y, z)=f(z, y, f(x, y, z))$ preserves $\{1,2,3\}$, in the second case $\widehat{g}$ does.

Claim 3.5. If $f \in[4,2,3 ; 2,1,4]$ or $f \in[4,2,3 ; 4,1,3]$ then $f$ is not minimal.

Proof. In the first case $f_{z}$ preserves $\{1,2,3\}$, in the second case $f_{y}$ does.

Claim 3.6. If $f \in[4,2,3 ; 2,4,3]$ then $f=M_{2}$.

Proof. Lemma 2.3 yields $\left.f\right|_{\langle 234\rangle}=p$ and $\left.f\right|_{\langle 324\rangle}=p$. Let us put $g(x, y, z)=$ $f(x, y, f(x, y, z))$. Then $g \in[f(1,2,4), 2,3 ; 2, f(1,3,4), 3]$. If none of $f(1,2,4)$, $f(1,3,4)$ equals 4 then $g$ preserves $\{1,2,3\}$. If one of them equals 4 , the other not, then $\widehat{g}$ is not minimal by Lemma 3.2 . So we must have $f(1,2,4)=f(1,3,4)=4$. In the same way we get $f(2,1,4)=f(3,1,4)=4, f(1,4,2)=f(1,4,3)=4$, etc. by using $g(x, y, z)=f(y, x, f(x, y, z)), f(x, f(x, y, z), y)$ etc.

Claim 3.7. If $f \in[4,2,3 ; 4,4,4]$ then $f$ is not minimal.

Proof. If $f_{z y}(2,1,3)=1$ then for $h(x, y, z)=f_{z y}\left(z, x, f_{z y}(x, y, z)\right)$ either $\widehat{h}$ preserves $\{1,2,3\}$ or fails to be minimal by Lemma 3.2 If $f_{z y}(2,1,3) \neq 1$ then the same holds for $f_{z y}$ itself, except when $f_{z y} \in[4,2,3 ; 2,4,3]$. In this case Claim 3.6 yields $f_{z y}=M_{2}$. We will see later that the clone generated by $M_{2}$ contains only three majority functions, and none of them equals $f$.

Theorem 3.8. Any minimal nonconservative majority function on A which satisfies $*$ is isomorphic to $M_{2}$ or it belongs to the set $S$.

Proof. Let $f$ be a function as stated in the theorem. According to Claim 3.3 and Claim 3.4 for every $a, b, c$ if neither $\left.f\right|_{\langle a b c\rangle}=p$ nor $\left.f\right|_{\langle a b c\rangle} \equiv u$ holds, then we must have that on two of the three triplets of $\langle a b c\rangle$ the value of $f$ equals the first variable, while on the third one $f$ equals $d$. If $f \notin S$ then this case really appears, so we can suppose (after an isomorphism if necessary) that $f(1,2,3)=4, f(2,3,1)=2$, $f(3,1,2)=3$. Now if $4 \notin f(\langle 213\rangle)$ then we get a contradiction by Lemma 3.2 If $\left.f\right|_{\langle 213\rangle} \equiv 4$ than Claim 3.7 implies that $f$ is not minimal. So $f(\langle 213\rangle)$ must be a three-element set containing 4 , and then again by Claims 3.3 and 3.4 we must have $f \in[4,2,3 ; 2,1,4], f \in[4,2,3 ; 4,1,3]$, or $f \in[4,2,3 ; 2,4,3]$. The first two of these is impossible by Claim 3.5 and in the third case Claim 3.6 shows that $f$ equals $M_{2}$.

\section{2.}

In this section we are going to search for the minimal functions of the set $S$. The conservative ones are already described, so we deal only with nonconservative functions. We assume $f$ to be such a function and we will prove several properties of $f$, until we find that only a few functions (essentially two) possess these properties, and these happen to be minimal. 
Definition 3.9. A ternary function $g$ is said to be cyclically commutative if it is invariant under the cyclic permutation of variables, i.e.

$$
g(x, y, z) \approx g(y, z, x) \approx g(z, x, y) .
$$

Claim 3.10. The function $f$ is cyclically commutative.

Proof. For contradiction, let us suppose $\left.f\right|_{\langle 124\rangle}=p$, and then by Lemma 3.2 we have also $\left.f\right|_{\langle 214\rangle}=p$. Since $f$ is not conservative, we may also suppose (without loss of generality) that $\left.f\right|_{\langle 123\rangle} \equiv 4$, and again by Lemma 3.2 we must have $\left.f\right|_{\langle 213\rangle} \equiv u$. First let us suppose $u \neq 4$. Then $f_{z y}$ preserves $\{1,2,3\}$ except when $f(3,1,2)=f(3,1,4)=f(3,4,2)=4$ or $f(3,1,2)=f(3,1,4)=f(3,4,1)=4$ and $f(3,4,2)=1$. In the first case $\widehat{f}_{z y} \in[1,2,4 ; u, u, u]$ so it is not a minimal function by Lemma 3.2 while in the second case $f_{z y} \in[1,1,4 ; u, u, u]$, hence $\widehat{f}_{z y}$ preserves $\{1,2,3\}$. If $u=4$ then we have also $f_{z y} \in[1,2,4 ; *, *, *]$ or $f_{z y} \in[1,1,4 ; *, *, *]$ or $f_{z y}(\langle 123\rangle) \subseteq\{1,2,3\}$. The first case is impossible, since then Theorem 3.8 implies that $\widehat{f}_{z y}$ is isomorphic to $M_{2}$, but then $f \notin\left[\widehat{f}_{z y}\right]$ shows that $f$ is not minimal. (In fact, the clone generated by $M_{2}$ contains no function from $S$ except for the first projection.) For $\langle 213\rangle$ we have also three possibilities: $f_{z y} \in[*, *, * ; 2,1,4]$, $f_{z y} \in[*, *, * ; 2,2,4]$ and $f_{z y}(\langle 213\rangle) \subseteq\{1,2,3\}$. The first one of these is impossible for the same reason as above. In the remaining cases $\widehat{f}_{z y}$ preserves $\{1,2,3\}$.

In the following we suppose $f$ to be a nonconservative cyclically commutative minimal majority function on $A$. In [3] these are determined by computer, here we give a straightforward description. We again suppose $\left.f\right|_{\langle 123\rangle} \equiv 4$, and $\left.f\right|_{\langle 213\rangle} \equiv u$. First we show that $f$ preserves $\{1,2,4\},\{1,3,4\}$ and $\{2,3,4\}$.

Claim 3.11. The only subset of $A$ not preserved by $f$ is $\{1,2,3\}$.

Proof. We separate two cases upon $u$.

Case 1. $\left.f\right|_{\langle 213\rangle} \equiv u \neq 4$. For contradiction let us suppose that $f$ does not preserve $\{1,2,4\}$. Then $\left.f\right|_{\langle 214\rangle} \equiv 3$ or $\left.f\right|_{\langle 124\rangle} \equiv 3$. In the first case $f\left(y, x, f(x, y, z)^{(2)}\right.$ preserves $\{1,2,3\}$ or $\{1,2,4\}$, in the second case $f\left(x, f_{z}(x, y, z), z\right)^{(2)}$ or $f_{z}$ preserves $\{1,2,3\}$ depending on whether $4 \in\{f(2,3,4), f(3,1,4)\}$ or not.

Case 2. $\left.f\right|_{\langle 213\rangle} \equiv 4$. What we have already proved of this claim means that if $\left.f\right|_{\langle a b c\rangle} \equiv d$ and $\left.f\right|_{\langle b a c\rangle} \equiv a$ then $f$ preserves the other three subsets of $A$, namely $\{a, b, d\},\{a, c, d\},\{b, c, d\}$. So if we again suppose that $f$ does not preserve $\{1,2,4\}$ then we must have $\left.f\right|_{\langle 124\rangle} \equiv 3$ and $\left.f\right|_{\langle 214\rangle} \equiv 3$. Similarly, $\left.f\right|_{\langle 234\rangle} \equiv 1$ if and only if $\left.f\right|_{\langle 324\rangle} \equiv 1$ and $\left.f\right|_{\langle 134\rangle} \equiv 2$ iff $\left.f\right|_{\langle 314\rangle} \equiv 2$. One can check that $f_{z}$ or $\widehat{f}_{z}$ preserves $\{1,2,3\}$ or $\{1,2,4\}$ except for only two functions (up to isomorphism and permutation of variables). For both of them $\left.f\right|_{\langle 134\rangle} \equiv 3$ and $\left.f\right|_{\langle 314\rangle} \equiv 4$, and for one we have $\left.f\right|_{\langle 234\rangle} \equiv 1$ and $\left.f\right|_{\langle 324\rangle} \equiv 1$, for the other one $\left.f\right|_{\langle 234\rangle} \equiv 4$ and $\left.f\right|_{\langle 324\rangle} \equiv 3$. In both cases $\widehat{f}_{z} \in[4,4,4 ; 3,3,3]$, hence by Case 1 it preserves $\{1,2,4\}$. We supposed that $f$ does not preserve this set, so $f \notin\left[\widehat{f}_{z}\right]$ and this contradicts the minimality of $f$.

We have proved that if $f \in S$ is a minimal function, then $f$ is cyclically commutative and preserves all but one three-element subsets of $A$. In the following two claims - as usually - we suppose that $f$ preserves $\{1,2,4\},\{1,3,4\},\{2,3,4\}$ and $\left.f\right|_{\langle 123\rangle} \equiv 4,\left.f\right|_{\langle 213\rangle} \equiv u$. Depending on whether $u=4$ or not, we will finally reach $M_{1}$ and $M_{3}$.

Claim 3.12. If $\left.f\right|_{\langle 213\rangle} \equiv u \neq 4$ then $\langle A ; f\rangle \cong\left\langle A ; M_{3}\right\rangle$.

Proof. We can suppose $\left.f\right|_{\langle 213\rangle} \equiv 3$ without loss of generality. We also suppose $\left.f\right|_{\langle 124\rangle} \equiv 4,\left.f\right|_{\langle 314\rangle} \equiv 4,\left.f\right|_{\langle 234\rangle} \equiv 4$, since otherwise $\widehat{f}_{z y}$ preserves $\{1,2,3\}$. For $g(x, y, z)=f(y, x, f(x, y, z))$ we have $g \in[f(1,2,4), f(3,2,4), f(1,3,4) ; 4,3,3]$. If none of $f(1,2,4), f(3,2,4), f(1,3,4)$ equals 4 , then $g^{(3)}$ preserves $\{1,2,3\}$. If there is a 4 amongst them, but 3 does not appear, then we put $h(x, y, z)=$ $f(g(x, y, z), g(z, y, x), g(x, z, y))$ and one calculate that the range of $h^{(2)}$ does not 
contain 4 , hence $f$ is not minimal by (2.3). Only nine functions remain; for two of them $g$ is isomorphic to $M_{3}$, hence $f$ is also. (The clone generated by $M_{3}$ contains only two functions from $S$, and only one of them can be equal to $f$.) If $g \in[2,4,3 ; 4,4,3]$ then $g(y, g(y, z, x), g(x, y, z))^{(2)}$ preserves $\{1,2,3\}$, if $g \in[1,3,4 ; 4,4,3]$ then $g(g(x, y, z), y, g(y, z, x))^{(2)}$ does so. In the remaining five cases $\{1,2,3\}$ is preserved by $f(g(x, y, z), g(z, x, y), g(y, z, x))$.

Claim 3.13. If $\left.f\right|_{\langle 213\rangle} \equiv 4$ then $\langle A ; f\rangle \cong\left\langle A ; M_{1}\right\rangle$.

Proof. Let $U=\{f(1,2,4), f(3,1,4), f(2,3,4)\}$ and $V=\{f(2,1,4), f(1,3,4)$, $f(3,2,4)\}$. If $U \neq\{4\}$ and $V \neq\{4\}$, then $\widehat{f}_{z y}$ preserves $\{1,2,3\}$. If $U=V=\{4\}$ then $f=M_{1}$. Now let us suppose $U=\{4\} \neq V$. If $4 \notin V$ then $\widehat{f}_{z y}$ is not minimal by Lemma 3.2 If this is not the case then by the previous claim $\widehat{f}_{z y}$ is isomorphic to $M_{3}$, but the clone $\left[M_{3}\right]$ contains no function which isomorphic to $f$. The case $V=\{4\} \neq U$ is similar.

3.3 .

We have now - up to isomorphism - only three functions: $M_{1}, M_{2}, M_{3}$, and these generate minimal clones.

Theorem 3.14. $M_{1}, M_{2}, M_{3}$ are minimal functions on $\{1,2,3,4\}$.

Proof. The proof is the same for all the three functions, so let $f$ be any of them. This function preserves the equivalence relation whose blocks are $\{1,4\},\{2\},\{3\}$, and its range does not contain the element 1. According to (1.1) and (2.3), the same is valid for an arbitrary majority function $g$ in $[f]$. These properties determine $\left.g\right|_{\{1,2,3\}}$ provided $\left.g\right|_{\{2,3,4\}}$ is given. Since $f$ preserves $\{2,3,4\}$ and $\left.f\right|_{\{2,3,4\}}$ is minimal, there exists an $h \in[g]$ such that $\left.h\right|_{\{2,3,4\}}=\left.f\right|_{\{2,3,4\}}$. Now $h$ has also the above mentioned two properties, so $\left.h\right|_{\{1,2,3\}}$ is uniquely determined: it can be nothing else than $\left.f\right|_{\{1,2,3\}}$. On $\{1,2,4\}$ and on $\{1,3,4\} f$ is constant 4 , consequently so are $g$ and $h$, hence $h=f$. Thus, for arbitrary $g \in[f], f \in[g]$ also holds, proving that $[f]$ is a minimal clone.

Remark. From the proof it is clear, that restriction to $\{2,3,4\}$ gives a one-to-one correspondence between the majority functions in $\left[M_{i}\right]$ and $\left[m_{i}\right]$. Hence these clones contain also 1,3 or 8 majority functions. They can be seen in the following table.

\begin{tabular}{|c|c|ccc|cccccccc|}
\hline & $M_{1}$ & $M_{2}$ & & & $M_{3}$ & & & & & & & \\
\hline$(1,2,3)$ & 4 & 4 & 2 & 3 & 3 & 3 & 4 & 3 & 4 & 4 & 3 & 4 \\
$(2,3,1)$ & 4 & 2 & 3 & 4 & 3 & 4 & 3 & 3 & 4 & 3 & 4 & 4 \\
$(3,1,2)$ & 4 & 3 & 4 & 2 & 3 & 3 & 3 & 4 & 4 & 4 & 4 & 3 \\
\hline$(2,1,3)$ & 4 & 2 & 4 & 3 & 4 & 3 & 4 & 4 & 3 & 4 & 3 & 3 \\
$(1,3,2)$ & 4 & 4 & 3 & 2 & 4 & 4 & 4 & 3 & 3 & 3 & 3 & 4 \\
$(3,2,1)$ & 4 & 3 & 2 & 4 & 4 & 4 & 3 & 4 & 3 & 3 & 4 & 3 \\
\hline$\{1,2,4\}$ & 4 & 4 & 4 & 4 & 4 & 4 & 4 & 4 & 4 & 4 & 4 & 4 \\
\hline$\{1,3,4\}$ & 4 & 4 & 4 & 4 & 4 & 4 & 4 & 4 & 4 & 4 & 4 & 4 \\
\hline$(4,2,3)$ & 4 & 4 & 2 & 3 & 3 & 3 & 4 & 3 & 4 & 4 & 3 & 4 \\
$(2,3,4)$ & 4 & 2 & 3 & 4 & 3 & 4 & 3 & 3 & 4 & 3 & 4 & 4 \\
$(3,4,2)$ & 4 & 3 & 4 & 2 & 3 & 3 & 3 & 4 & 4 & 4 & 4 & 3 \\
\hline$(2,4,3)$ & 4 & 2 & 4 & 3 & 4 & 3 & 4 & 4 & 3 & 4 & 3 & 3 \\
$(4,3,2)$ & 4 & 4 & 3 & 2 & 4 & 4 & 4 & 3 & 3 & 3 & 3 & 4 \\
$(3,2,4)$ & 4 & 3 & 2 & 4 & 4 & 4 & 3 & 4 & 3 & 3 & 4 & 3 \\
\hline
\end{tabular}




\section{REFERENCES}

[1] B. Csákány, All minimal clones on the three-element set, Acta Cybernet. 6 (1983), no. 3, 227-238.

[2] B. Csákány, On conservative minimal operations, Lectures in Universal Algebra (Szeged, 1983), Colloq. Math. Soc. János Bolyai, 43, North-Holland, Amsterdam, 1986, 49-60.

[3] Z. Csibor: Majority functions, Masters Thesis (Szeged, 1988) (In Hungarian).

[4] E. Post, The two-valued iterative systems of mathematical logic, Annals of Mathematics Studies, no. 5, Princeton University Press, Princeton, 1941.

[5] R. W. Quackenbush: Some remarks on categorical algebras, Algebra Universalis 2 (1972), 246.

[6] R. W. Quackenbush: A survey of minimal clones, Aequationes Mathematicae 50 (1995), 3-16.

[7] I. G. Rosenberg, Minimal clones I. The five types, Lectures in Universal Algebra (Szeged, 1983), Colloq. Math. Soc. János Bolyai, 43, North-Holland, Amsterdam, 1986, 405-427.

[8] B. Szczepara, Minimal clones generated by groupoids, Ph.D. Thesis, Université de Montréal, 1995.

[9] Á. Szendrei, Clones in Universal Algebra, Séminaire de Mathématiques Supérieures, 99, Presses de L’Université de Montréal, 1986.

Bolyai Institute, University of Szeged, Aradi vértanúk tere 1, H6720, Szeged, HunGARY

E-mail address: twaldha@math.u-szeged.hu 